\title{
5G F-OFDM Waveform Based Software-Defined Radio Technology
}

\author{
Dia Mohamad Ali ${ }^{1, *}$, Zhraa Zuheir Yahya ${ }^{2}$ \\ ${ }^{1}$ College of Electronics Engineering, Ninevah University, Ninevah, Iraq \\ ${ }^{2}$ Department of Communication Engineering, Ninevah University, Ninevah, Iraq \\ Received 10 November 2021; received in revised form 16 December 2021; accepted 17 December 2021 \\ DOI: https://doi.org/10.46604/peti.2022.8887
}

\begin{abstract}
Filtered-orthogonal frequency division multiplexing (F-OFDM) is a quasi-orthogonal waveform candidate for the applications of the fifth generation $(5 \mathrm{G})$ communication system. In this study, an F-OFDM waveform with unequal sub-band sizes is proposed to improve the spectrum efficiency (SE) of the 5G system. The proposed waveform is modeled with the Blackman window-sinc filter and is developed based on the software-defined radio (SDR) technology for practical implementation. The result shows that the F-OFDM performance of the simulation and hardware implementation is approximately the same. The SE using the proposed F-OFDM waveform is $6 \%$ and $5.8 \%$ higher than the SE using the conventional OFDM waveform under the simulation in the LabVIEW NXG simulator and under the practical use in the universal software radio peripheral (USRP) platform, respectively.
\end{abstract}

Keywords: filtered-orthogonal frequency division multiplexing, spectrum efficiency, software-defined radio, sub-band, fifth generation

\section{Introduction}

The fifth generation $(5 \mathrm{G})$ communication system has been designed to satisfy the demand generated from the exponential growth in the number of connected devices, the variety of hungry data rate services, as well as the shortcomings of the fourth generation $(4 \mathrm{G})$ technologies [1]. To achieve the goal and offer a diverse set of services over the available spectrum, the 5G system must be versatile and adaptive to the developed technologies [2]. The choice of physical layer waveform plays an important role in determining the system performance.

A new, flexible, and adaptive waveform design is required for the $5 \mathrm{G}$ system. The new waveform should support massive machine type communication (mMTC), enhanced mobile broadband (eMBB), and ultra-reliable and low latency communications (URLLC) for a wide range of frequencies. Furthermore, the newly adopted waveform is capable of dealing with the multi-antenna system containing hundreds of steerable antenna elements. The variety of waveform numerology designs (i.e., subcarrier spacing, cyclic prefix (CP), and transmission time interval) must be taken into account depending on the deployment options and the operation frequency. The new waveform allows different numerologies to be mixed on the used carrier frequency, where the subcarriers of specified numerology interfere in the frequency domain with other subcarriers of another numerology.

In the $4 \mathrm{G}$ system, orthogonal frequency division multiplexing (OFDM) is a promised waveform for sustaining the spectrum efficiency (SE), which allocates $10 \%$ of the spectrum as a guard band [3]. However, OFDM suffers from higher sidelobes due to the rectangular pulse shape in the time domain (which limits the desired SE for 5G applications), the high peak-to-average power ratio (PAPR), and the strictness of synchronization. Furthermore, with the ever-increasing use of

* Corresponding author. E-mail address: Dia.ali@uoninevah.edu.iq

Tel.: 9647701650111 
wireless communication services and the growing number of users, a great deal of attention has been given to SE (to minimize the guard band), especially at the low- and mid-operation frequency bands for broadband experience and capacity enhancement. For these reasons, OFDM is not recommended for the $5 \mathrm{G}$ system, and new waveforms are candidates for improving the performance.

The most important waveforms that have been proposed for the 5G system are filtered-OFDM (F-OFDM), universal filter multicarrier (UFMC), generalized frequency division multiplexing (GFDM) [4], and filter bank multicarrier (FBMC) [5]. In UFMC, a group of orthogonal subcarriers is filtered using the same or different filter performance in each sub-band to minimize the out-of-band emission (OOBE). A zero prefix (ZP) is added to mitigate the effect of inter-symbol interference (ISI). The filter length in UFMC must be within the ZP length [6]. The main limitation of UFMC is that it is not recommended for applications that require higher data rates [7]. GFDM is a non-orthogonal waveform with block base transmission, in which the subcarriers are spaced with more flexibility and the pulse shaping is performed on the subcarriers for eliminating inter-carrier interference (ICI) and providing spectral confinement. In addition, the CP insertion lessens the impact of ISI. GFDM is preferred for services with lower data rates [8]. The more complex processing required at the receiver makes it inefficient for low latency applications in 5G [1].

FBMC is a non-orthogonal waveform. The filtering is performed per subcarrier, thus a long filter length is used. The accomplished SE using FBMC is higher than that using other waveforms due to the advanced filter design. Therefore, CP can be omitted. Offset quadrature amplitude modulation (OQAM) is used to preserve time-domain signals. FBMC is not preferred for $5 \mathrm{G}$ services because the length of the filter requires more processing and hence increases the latency [8]. In addition, the complexity is increased with the multi-input multi-output (MIMO) technology [9]. In general, F-OFDM is the most suitable waveform for $5 \mathrm{G}$ compared to other waveforms because of its lower complexity, and it offers the advantages of OFDM as well as greater SE achievement due to the utilized filter. F-OFDM is more adaptable, requires a simple equalizer at the receiver, and can be compatible with the MIMO technology. It does not require synchronized transmission, thus supporting multi-services, and it offers forward and backward compatibility. Additionally, it can coexist with other waveforms [10].

The software-defined radio (SDR) technology is a revolutionary wireless communication system that enables the system to be completely reprogrammed and reconfigured via software modules, except for the radio frequency (RF) stage. For the first time in the real world, the universal software radio peripheral (USRP) platform assists in the design, development, and prototyping of the next generation of wireless system technologies in a high-quality and timely manner. The USRP platform has two partitions: a fixed motherboard and a plug-in daughterboard. An analog-to-digital converter (ADC) and a digital-to-analog converter (DAC) are included on the motherboard. In addition, a host personal computer (PC) interface and a field-programmable gate array (FPGA) for digital down-conversion (DDC) with a programmable decimation rate have been included. The daughterboard is in charge of the basic RF front-end functionality. SDRs are well-suited for a variety of applications due to their wide bandwidths and adaptable RF front ends.

The USRP platform is designed for RF applications operating from DC to $6 \mathrm{GHz}$, including spectrum monitoring, cognitive radio, mobile phones, satellite navigation, and intelligent radios. Users can use the USRP products in commercial, academic, and military applications with flexible and reusable field deployments [11]. It is possible to use SDRs to perform different waveforms to analyze the performance in real-time, which allows the implementation of many studies that would otherwise be only by simulation [12].

In this work, F-OFDM is modeled with four different sub-band sizes based on the Blackman window-sinc filter to test the performance in simulation using LabVIEW NXG and in practice using the SDR technology. The SE enhancement using F-OFDM and OFDM is compared. The proposed F-OFDM waveform is presented by optimizing the guard band to the minimum between the sub-bands so that the waveform can meet the $5 \mathrm{G}$ requirements. 


\section{Literature Review}

Zhang et al. [10] described the general construction of F-OFDM with significant design aspects such as the filter design and guard tone. A comparison of waveform contenders for $5 \mathrm{G}$ was also included in the study demonstrating the merits of F-OFDM. The simulation model with four different kinds of services indicates that F-OFDM offers $46 \%$ higher throughput gains than OFDM. Abdoli et al. [6] proposed an F-OFDM waveform to attain the required spectrum localization while keeping a permissible limit of ISI and ICI. The proposed F-OFDM model enables the asynchronous transmission of filtered orthogonal frequency division multiple access (F-OFDMA) / filtered-discrete Fourier transform-spread OFDMA (F-DFT-S-OFDMA) at each transmitter with a spectrum shaping filter to minimize the sidelobe leakage, and the receiver employs a bank of filters to reject inter-user interference (IUI).

Wang et al. [4] demonstrated the performance of three key technologies for the 5G system: sparse code multiple access (SCMA), polar codes, and F-OFDM, based on the field trial testbed developed by NTT DOCOMO and HUAWEI. The results show that F-OFDM improves the performance in terms of throughput and SE by 7.47\%. Yang et al. [13] proposed an F-OFDM waveform with two sub-bands employing the Nuttall-Blackmanharris window filter as a candidate waveform for 5G. Various window functions were used to evaluate the F-OFDM performance. The results of the simulation were compared to the Hamming window and OFDM. It is observed that, as compared to OFDM, their proposed filter scheme has the lower OOBE and the same bit error rate (BER). Gerzaguet et al. [14] introduced a block filtered-OFDM (BF-OFDM) waveform to support asynchronous multi-user connections and spectrum localization. The results of the proposed waveform offered the same performance in terms of SE and BER compared to the conventional CP-OFDM waveform with the complexity cost and simple equalizer at the receiver.

Yang et al. [15] proposed an F-OFDM waveform with two sub-bands (users) in uplink asynchronous transmission to achieve the BER objective and SE enhancement using a window-sinc filter. For various numerologies between the users, the authors derived the theoretical computation of BER on ISI, ICI, and IUI. The filter parameter and guard band were optimized to enhance the performance and outperform the conventional OFDM waveform. Chandran et al. [16] proposed an index modulation F-OFDM waveform with two sub-bands of different parameters to combat the effect of inter-numerology interference (INI) between the sub-bands. The obtained simulation results show that the performance of the mixed numerology waveform improves in terms of BER in comparison to the conventional OFDM waveform due to the reduced interference.

\section{USRP-X310}

USRP-X310 is the third generation of the USRP device developed by Ettus Research LLC., which offers high performance and expandable SDR platforms for modeling and prototyping the next generation wireless communication systems. The device combines multiple RF daughterboard slots with high bandwidth, which cover the DC-6 GHz range of frequencies and the baseband bandwidth of $160 \mathrm{MHz}$. Supplied with the ultra-high-speed interfaces PCIe and dual 10/1 GigE, the device has the largest customizable kintex-7 FPGA. USRP-X310 can be used for advanced wireless system prototyping in Wi-Fi, cellular applications, multiple antennas (MIMO) testbeds, passive radar systems, and some intelligence signals [17]. Fig. 1 is the photograph of USRP-X310. The internal components of USRP-X310 are shown in Fig. 2.

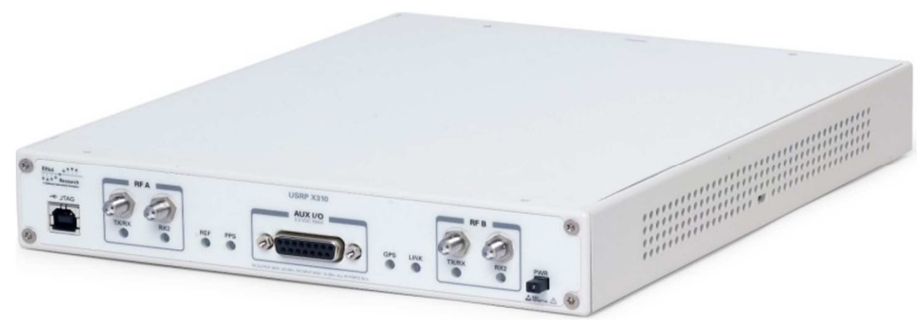

Fig. 1 USRP-X310 


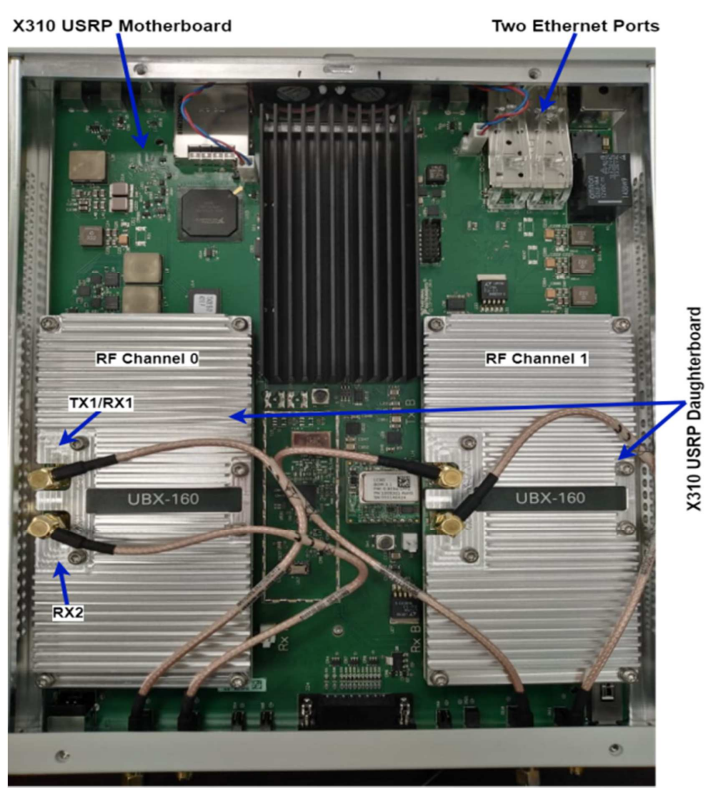

Fig. 2 Architecture of USRP-X310

\section{Structure of F-OFDM}

On the transmitting side, the entire band is split into smaller sub-bands where the data in each sub-band is processed separately. The bitstreams for each sub-band are mapped to various quadrature amplitude modulation (QAM) constellation symbols. The symbols are transformed from serial to parallel, and the inverse fast Fourier transform (IFFT) is performed at each sub-band to obtain the orthogonality in which the subcarrier is produced at the zero-crossing point of the other subcarrier to avoid interference. To mitigate the impact of multipath propagation, dissimilar lengths of CP are inserted for the sub-bands. The signal is then filtered by a digital prototype finite impulse response (FIR) filter to provide the isolation between numerology-based sub-bands. Finally, the F-OFDM signal of all sub-bands is combined to be transmitted over the channel.

The inverse processes of the transmitter are carried out on the receiving side. The signal is filtered to take the desired sub-band spectrum and attenuate the others with a filter designed as a matched copy of the transmission filter. A simple equalization process necessitates canceling the effects of the channel and the proposed filter. CP is removed and fast Fourier transform (FFT) is performed on the desired sub-band to be able to demodulate the received data. Fig. 3 illustrates the F-OFDM transceiver as a block diagram.
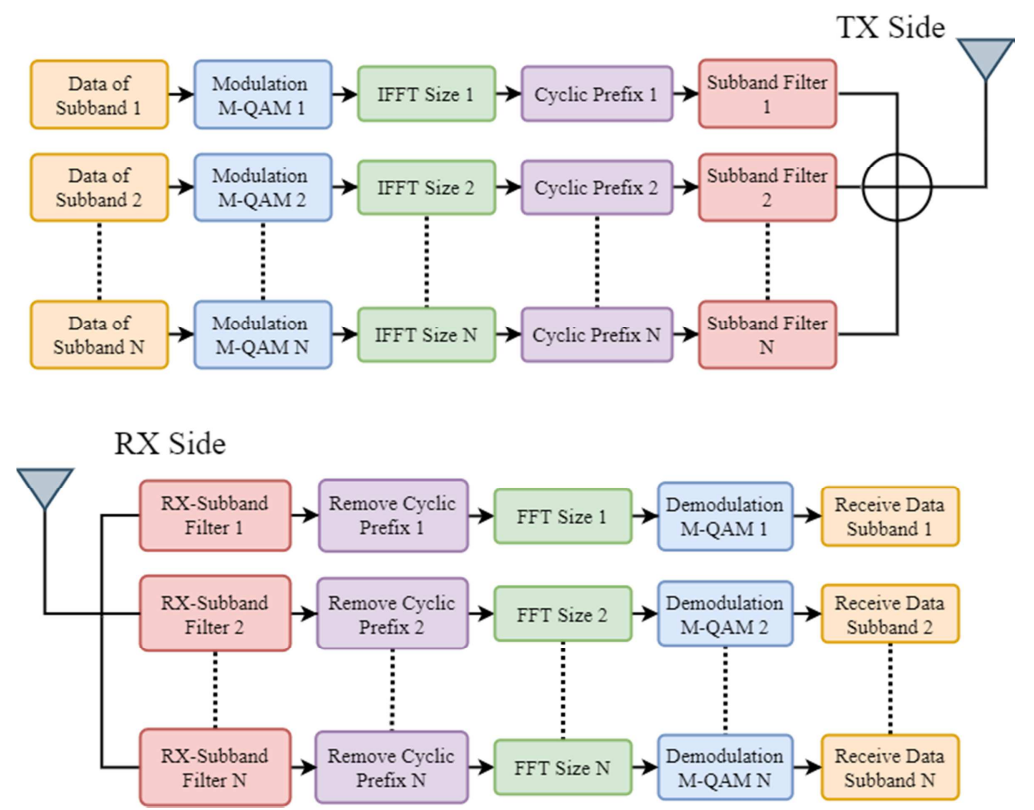

Fig. 3 Structure of the F-OFDM transceiver 
If $\left\{x_{m, k}\right\}_{k=0}^{N-1}$ is a modulated data symbol transmitted at the $m$ th OFDM symbol, then for each sub-band the OFDM signal can be expressed as:

$$
x_{m}(t)=\sum_{k=0}^{N-1} x_{m, k} e^{j 2 \pi k \Delta f t} ; 0 \leq t \leq T_{s}
$$

where $T s, \Delta f$, and $N$ are the symbol duration, the subcarrier space, and the number of subcarriers of OFDM signals, respectively. At the receiver, the transmitted symbol can be detected if there is no channel distortion.

$$
x_{m, k}=\frac{1}{T_{s}} \int_{0}^{T s} x_{m}(t) e^{-j 2 \pi \Delta f t}
$$

A discrete-time description of the transmitted signal can be obtained by sampling the continuous signal at $t=T_{s} / N$. Eq. (1) can be expressed as:

$$
x_{m}\left(n \frac{T s}{N}\right)=\sum_{k=0}^{N-1} x_{m, k} e^{j 2 \pi \frac{k n}{N}}
$$

where $n$ is the discrete sampling points. The signal represents the inverse discrete Fourier transform (IDFT) of the transmitted symbol and can be efficiently calculated by IFFT. At the receiver, it can demodulate the signal by FFT instead of integration. When CP is inserted at the beginning of the OFDM symbol with the time $T_{C P}$, the OFDM signal time interval is extended to $T$ $=T_{C P}+T_{s}$, then the transmitted signal $\dot{\mathrm{x}}_{m}(t)$ is expressed as:

$$
\dot{x}_{m}(t)=\sum_{k=0}^{N-1} x_{m}, k e^{j 2 \pi k \Delta f t} ;-T_{C P} \leq t \leq T_{s}
$$

At the receiver, the signal can be written as:

$$
y_{m, k}=H_{k} \dot{x}_{m, k}+Z_{k}
$$

where $H_{k}$ is the channel frequency response and can be represented by:

$$
H_{k}=\sum_{i} \gamma_{i} e^{-j 2 \pi k \Delta f \tau i}
$$

where $\tau_{i}$ and $\gamma_{i}$ are the delay and the complex amplitude of the $i$ th path, respectively. $Z_{k}$ is the impact of additive white Gaussian noise (AWGN).

The F-OFDM signal for each sub-band $\ddot{x}_{l}(n)$ is obtained by the linear convolution of the filter impulse response with the OFDM signal for that sub-band:

$$
\ddot{x}_{l}(n)=\dot{x}_{l}(n) \times f_{l}(n)
$$

where $\dot{\mathrm{x}}_{l}(n)$ is the OFDM signal for the $l$ th sub-band with the CP samples, and $f_{l}(n)$ is the impulse response of the digital filter of the $l$ th sub-band. The received signal is filtered and has an impulse response given by $f_{l}(-n)$, which is a matched copy of the transmission filter.

The filter must meet certain criteria to accommodate the $5 \mathrm{G}$ waveform requirements. The passband of the designed filter should be as flat as possible to ensure that the distortion of the filter does not affect the data subcarriers; particularly, the sub-band edge subcarriers should be kept to a minimum. The roll-off of the filter should begin at the edges of a passband and a 
very narrow transition band. This ensures that the system bandwidth is used as efficiently as possible with the minimum effort of guard band overhead. Furthermore, neighboring sub-band signals with various numerologies can be placed in the frequency domain next to each other with a minimum number of guard bands. Higher-order filters will reduce the OOBE by decreasing the sidelobes. Usually, the filter length exceeds the CP length for better localization in the frequency domain. The Blackman window-sinc filter designed in Matlab and interfaced to the LabVIEW is applied into the F-OFDM sub-bands. The magnitude and phase response of the used filter are illustrated in Fig. 4.

The normalized sinc function prototype filter $f_{d}(n)$ is represented in the equation below:

$$
f_{d}(n)=\operatorname{sinc}\left[\left(N_{\text {sub }}+2 \times \text { toneoffset }\right) \times \frac{n}{\text { FFTsize }}\right]
$$

where $N_{\text {sub }}$ is the number of used subcarriers for each sub-band, and toneoffset is an increased value specified at each side of the sub-band filter.

The FIR window-sinc filter for the $l$ th sub-band is obtained after multiplying the sinc function by a time-domain window function $w(n)$, as follows:

$$
f_{l}(n)=f_{d}(n) \cdot w(n)
$$

For the Blackman window, $w(n)$ can be defined as the equation below [18]:

$$
w(n)=a_{o}-a_{1} \cos \left(\frac{2 \pi n}{N}\right)+a_{2} \cos \left(\frac{4 \pi n}{N}\right)
$$

where $a_{o}=0.42, a_{1}=0.5$, and $a_{2}=0.08$.

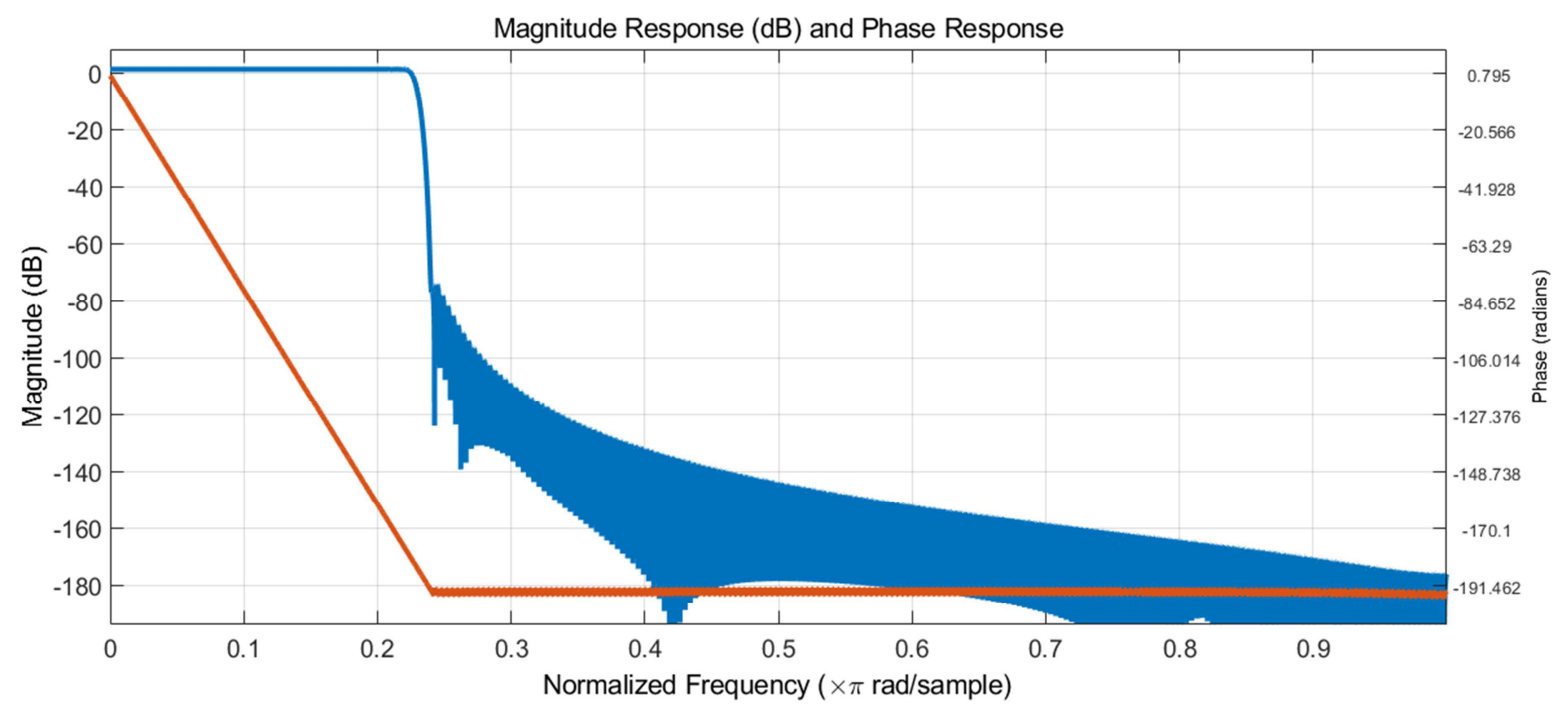

Fig. 4 Magnitude and phase response of the utilized digital filter

\section{Simulation Results and Analysis}

The waveform is designed to fragment the allocated spectrum into unequal sub-band sizes using LabVIEW NXG simulator. Each sub-band has a different number of data subcarriers and a different QAM order to match the multi-service requirements for $5 \mathrm{G}$. The simulation parameters are presented in Table 1. 
Table 1 F-OFDM simulation parameters

\begin{tabular}{|c|c|}
\hline Parameter & Setting \\
\hline Filter type & Window-sinc filter \\
\hline IFFT/FFT & 1024 \\
\hline Filter order & 513 \\
\hline Modulation type/order & QAM/16,64,128,256 \\
\hline CP length & 64 samples/sub-bands \\
\hline Subcarrier spacing & $15 \mathrm{kHz}$ \\
\hline Sub-band with unequal bandwidth & $13.8 \mathrm{MHz}$ \\
\hline Window function & Blackman \\
\hline Number of data subcarriers per sub-band & $372,186,93$ \\
\hline
\end{tabular}

\subsection{Simulation analysis}

The whole allocated spectrum is divided into four unequal sub-bands, having unequal IFFT/FFT sizes and diversity of QAM orders. The Blackman filter is applied to each sub-band to suppress the OOBE and optimize the guard band between the sub-bands. Figs. 5 and 6 illustrate the power spectrum of the four sub-bands based on the parameters of Table 1 in LabVIEW NXG for OFDM and F-OFDM, respectively. It can be seen that F-OFDM can reduce the sidelobes and minimize the guard band. The combination of the OFDM sub-bands results in the $-30 \mathrm{~dB}$ reduction in sidelobes, whereas the combination of F-OFDM sub-bands results in the $-200 \mathrm{~dB}$ reduction in sidelobes.

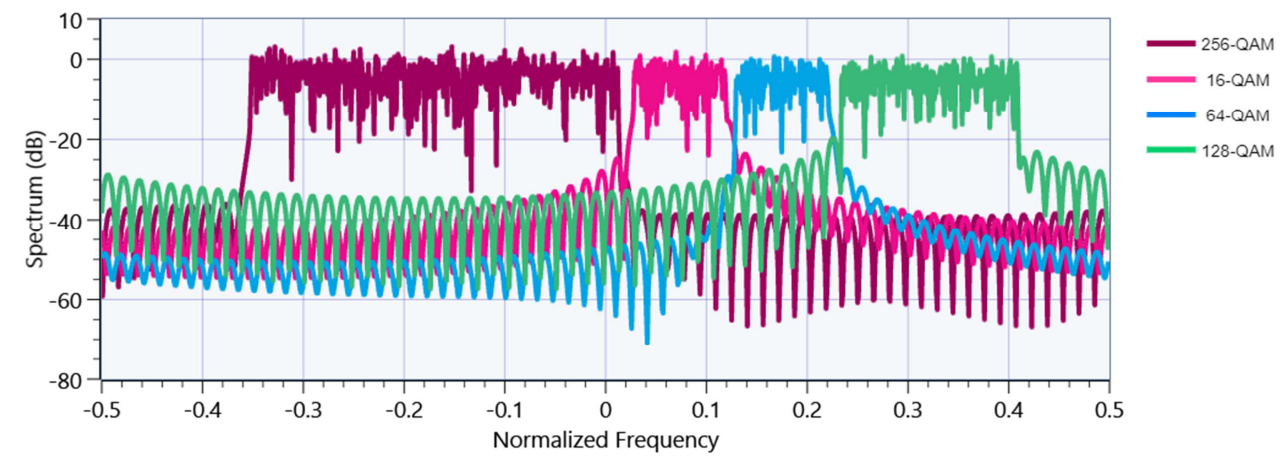

Fig. 5 The OFDM power spectrum with four unequal sub-band sizes in LabVIEW NXG

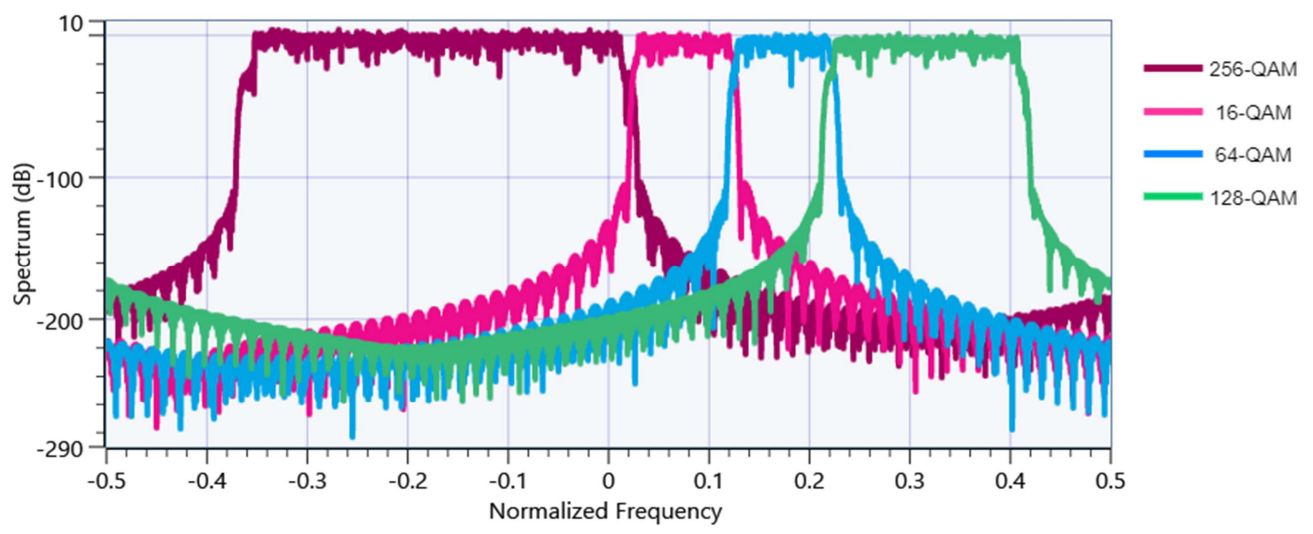

Fig. 6 The F-OFDM power spectrum with four unequal sub-band sizes in LabVIEW NXG

The SE performance of the designed waveform is compared to that of the OFDM-based AWGN channel. The calculated SE based equation is expressed as follows [15]:

$$
S E=\frac{\sum_{s u b=1}^{S U B} \sum_{n=n u-1}^{N s u b+n_{u-1}} C_{s u b, n}}{\sum_{s u b=1}^{S U B} B W_{s u b}+\sum_{i=1}^{S U B-1} B W_{g, i}}
$$


where $S U B$ is the total number of sub-bands, and $n_{u}$ is the location of the active subcarrier for each sub-band. $C_{s u b, n}$ is the capacity of each sub-band, which is measured in bit/s and calculated based on the properly received bits per simulation time. $B W_{\text {sub }}$ represents the utilized bandwidth in each sub-band, which is calculated by multiplying the number of used data subcarriers in each sub-band by the subcarrier spacing. $B W_{g, i}$ represents the guard band between the sub-bands, which is calculated based on the number of unused tones in each sub-band multiplied by the subcarrier spacing. The optimizing guard band is obtained by offsetting the sub-bands from each other with the steps equal to the subcarrier spacing until the minimum guard is obtained.

Fig. 7 illustrates the obtained normalized SE of unequal sub-band sizes. At lower values of Eb/No, the SE enhancement achieves 4.7\%; at higher Eb/No values, the SE enhancement is approximately 6\%. Fig. 8 shows the average BER performance of the F-OFDM compared to that of OFDM. It can be seen that the average BER for F-OFDM is approximately the same as that for OFDM. In Fig. 9, the comparison between the PAPR of F-OFDM and OFDM is presented. It can be noticed that F-OFDM has a higher PAPR. This increase lies in that the used filter widens the power distribution among the samples, causing an increase in the gap between the mean and the peak power values and thus leading to an increase in PAPR.

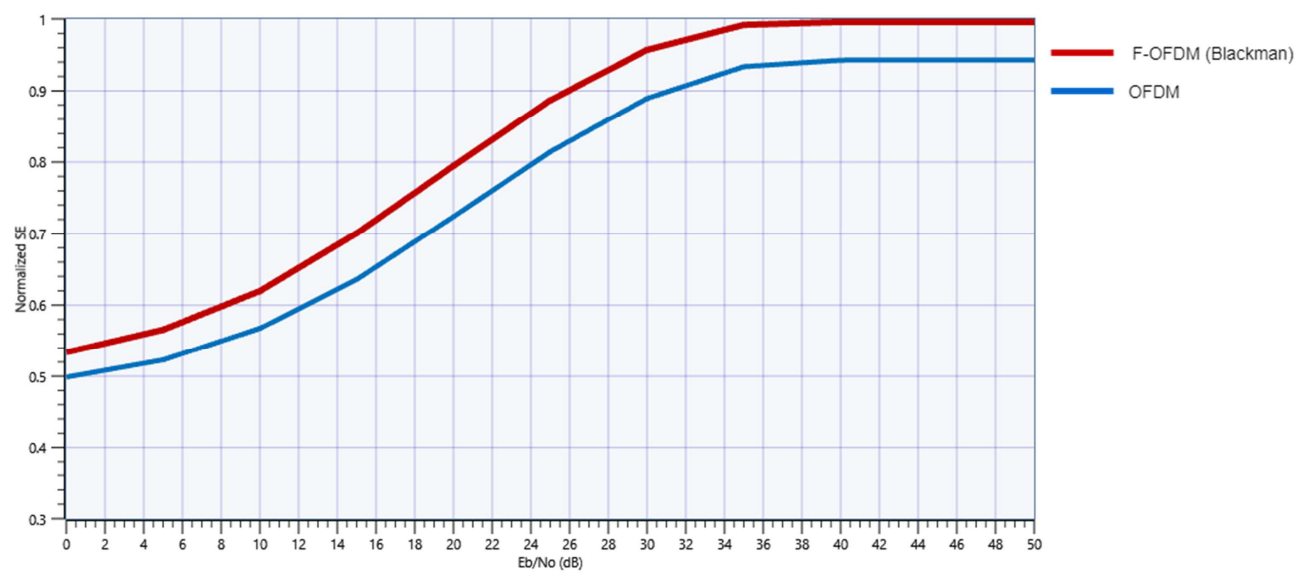

Fig. 7 Normalized SE of the F-OFDM with unequal sub-band sizes

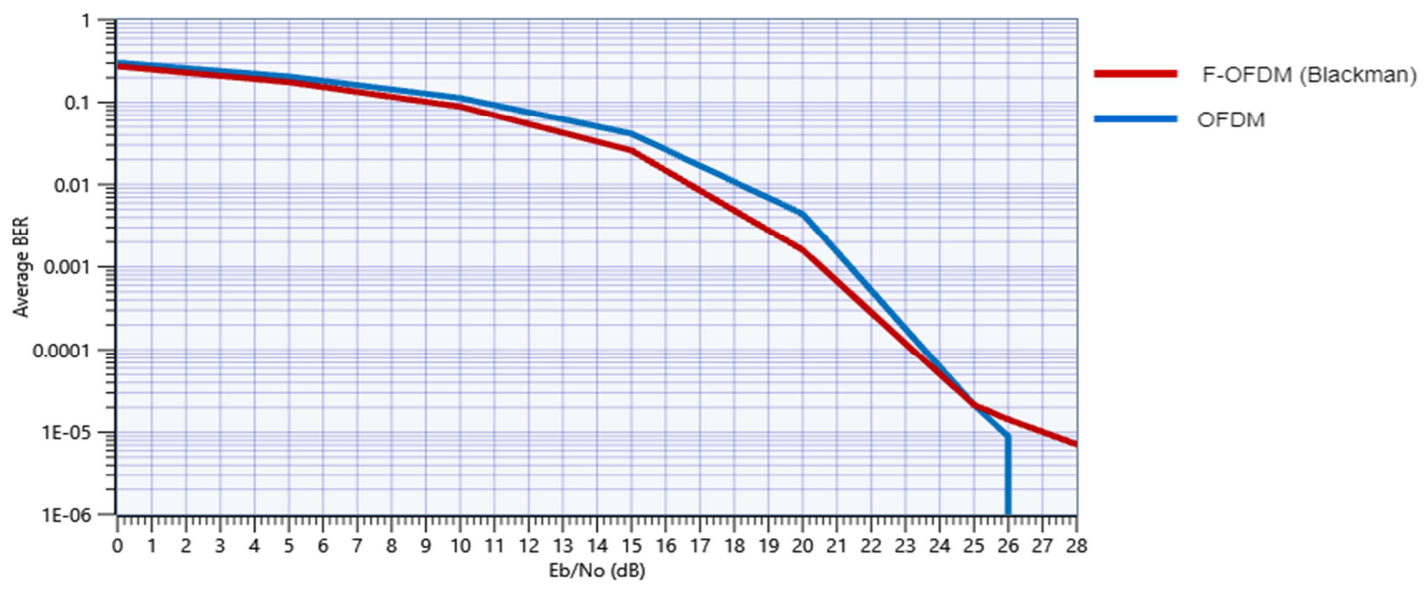

Fig. 8 Average BER of F-OFDM and OFDM

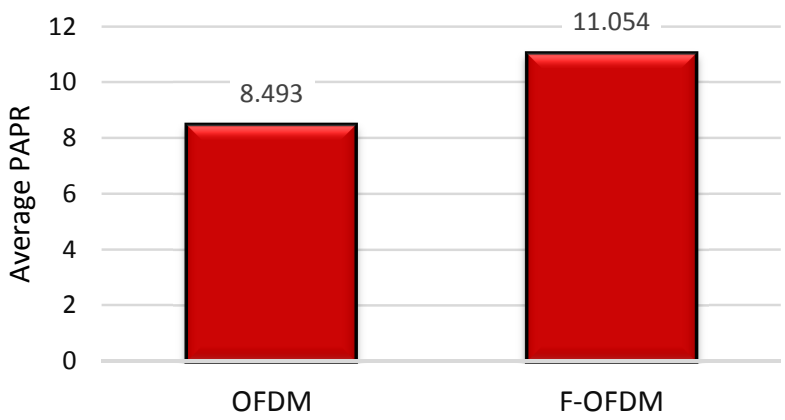

Fig. 9 Average PAPR of F-OFDM and OFDM 


\subsection{Testbed of the F-OFDM sub-band waveform}

In the proposed testbed, USRP-X310 is used with two RF chains programmed through LabVIEW NXG, and is specialized for simulating and testing communication systems. This study tests the designed waveform in real-time by implementing the transmitted signal of the designed waveform at $915 \mathrm{MHz}$ carrier frequency through a total bandwidth of 15.36 MHz with $20 \mathrm{~dB}$ transmission gain. The proposed testbed is shown in Fig. 10, where the waveform is processed on the PC and connected to USRP-X310 through an Ethernet cable. USRP-X310 transmits the up-converted signal to the air interface through a log-periodic antenna. At the receiving end, the signal is received through the second antenna that is present on the receiving chain of the same USRP and down-converted. The down-converted signal is converted to digital form to be processed on the PC.

In this work, the signal to be transmitted is free of reference signals for synchronization and channel estimation, which needs to be studied in certain algorithms that are currently outside the scope of research. To achieve the synchronization between the transmitting and receiving nodes, the signals are configured by the niUSRP configure time start trigger node on both transmitting and receiving sides. Because of the multipath phenomenon, the signal distorts (phase and amplitude) while being transmitted over the channel. To compensate for this distortion at the receiver, an estimator for channel characteristics is required. The equalizer is built on the basis of finding the estimated transfer function of the channel by sending the information known by both the sender and receiver. The equalization is simply achieved by multiplying the received signal with the transfer function of the channel to complete the receiver process. The hardware setting parameters for the USRP device are presented in Table 2. The summary of the transmitting and receiving processes using the USRP device is illustrated in Fig. 11.

Figs. 12 and 13 show the transmitted power spectrum of the unequal-sized waveform and the constellation points in F-OFDM and OFDM, respectively. In comparison to OFDM, the F-OFDM spectrum achieves higher sidelobe suppression and a lower guard band. Figs. 14 and 15 show the equalized received spectrum and constellation points for F-OFDM and OFDM, respectively. Because of the transmission over the noisy channel, there is a minor change in the received spectrums of F-OFDM and OFDM. According to the constellation diagrams, F-OFDM, like OFDM, supports the same characteristics. After equalizing the signal due to the channel effect, the received constellation diagrams in the proper form are obtained and the same transmission data is observed.

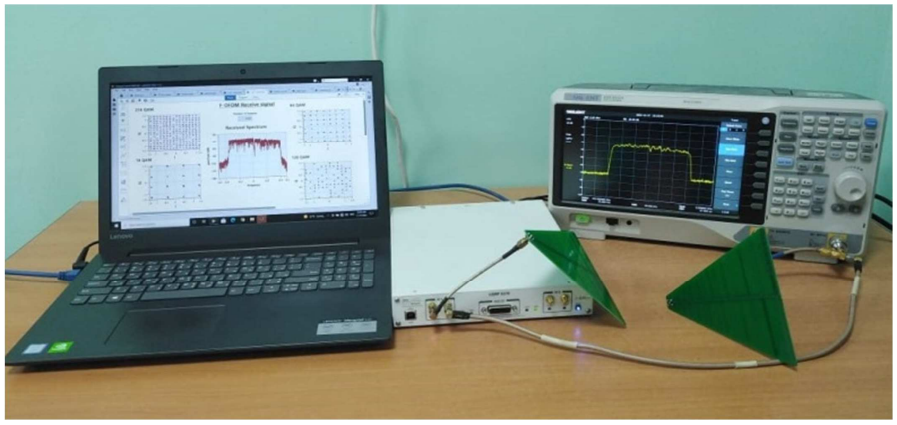

Fig. 10 Testbed for the F-OFDM sub-band waveform

Table 2 Transmitting/receiving setting parameters for USRP

\begin{tabular}{|c|c|c|}
\hline Parameter & Transmitting setting & Receiving setting \\
\hline Device name & 192.168 .10 .2 & 192.168 .10 .2 \\
\hline $\begin{array}{c}\text { In-phase and quadrature } \\
\text { component (IQ) rate }\end{array}$ & $15.36 \mathrm{MHz}$ & $15.36 \mathrm{MHz}$ \\
\hline Carrier frequency & $915 \mathrm{MHz}$ & $915 \mathrm{MHz}$ \\
\hline Active channel & 0 & 0 \\
\hline Active antenna & $\mathrm{TX} 1$ & $\mathrm{RX} 2$ \\
\hline Gain & 20 & 1 \\
\hline
\end{tabular}




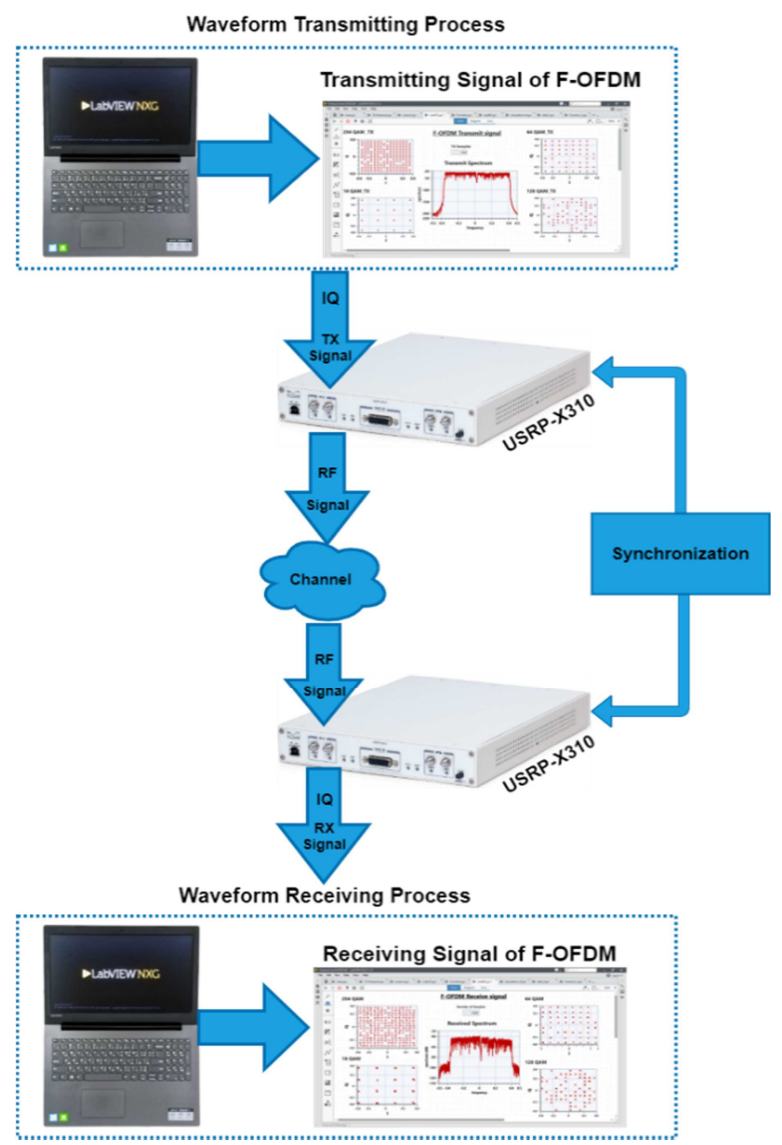

Fig. 11 Transmitting/receiving process for the F-OFDM with unequal sub-band sizes

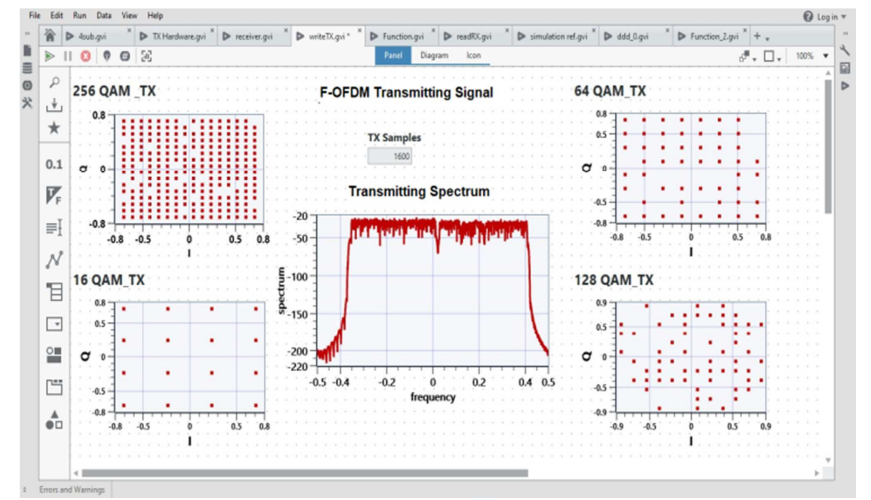

Fig. 12 F-OFDM signals after the transmitting process

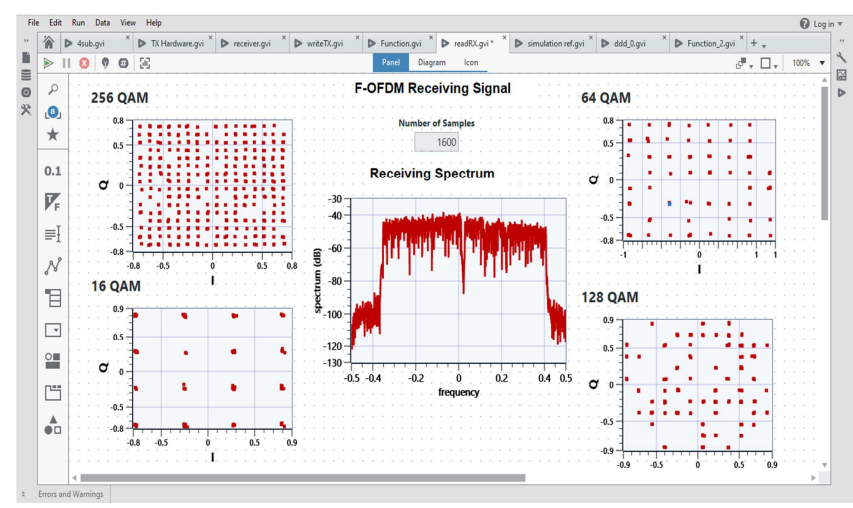

Fig. 14 F-OFDM signals after the receiving process

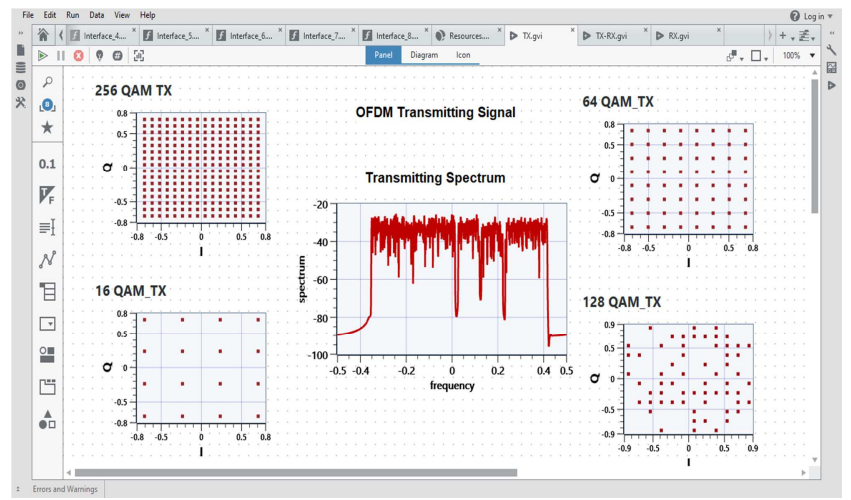

Fig. 13 OFDM signals after the transmitting process

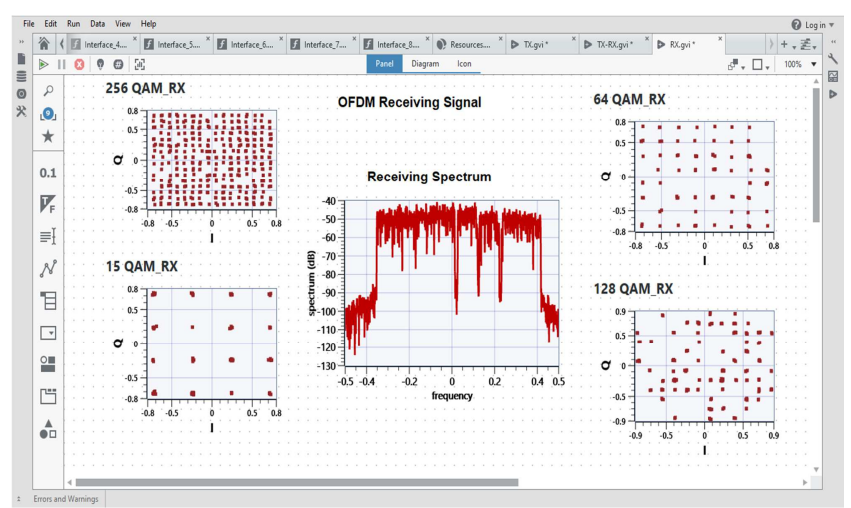

Fig. 15 OFDM signals after the receiving process

Figs. 16 and 17 illustrate the real received power spectrum of F-OFDM and OFDM, respectively. It can be seen that the proposed F-OFDM-based Blackman window-sinc filter achieves the higher suppression of sidelobes and thus the lower guard band capability. To compare the SE performance under the simulation and real environment, Fig. 18 illustrates the obtained SE 
enhancement over OFDM in practice. It is obvious that the SE enhancement of the proposed waveform under the real environment achieves approximately $5.8 \%$, which is similar to that under the simulation environment. Table 3 compares the F-OFDM waveforms proposed in previous studies and this study.

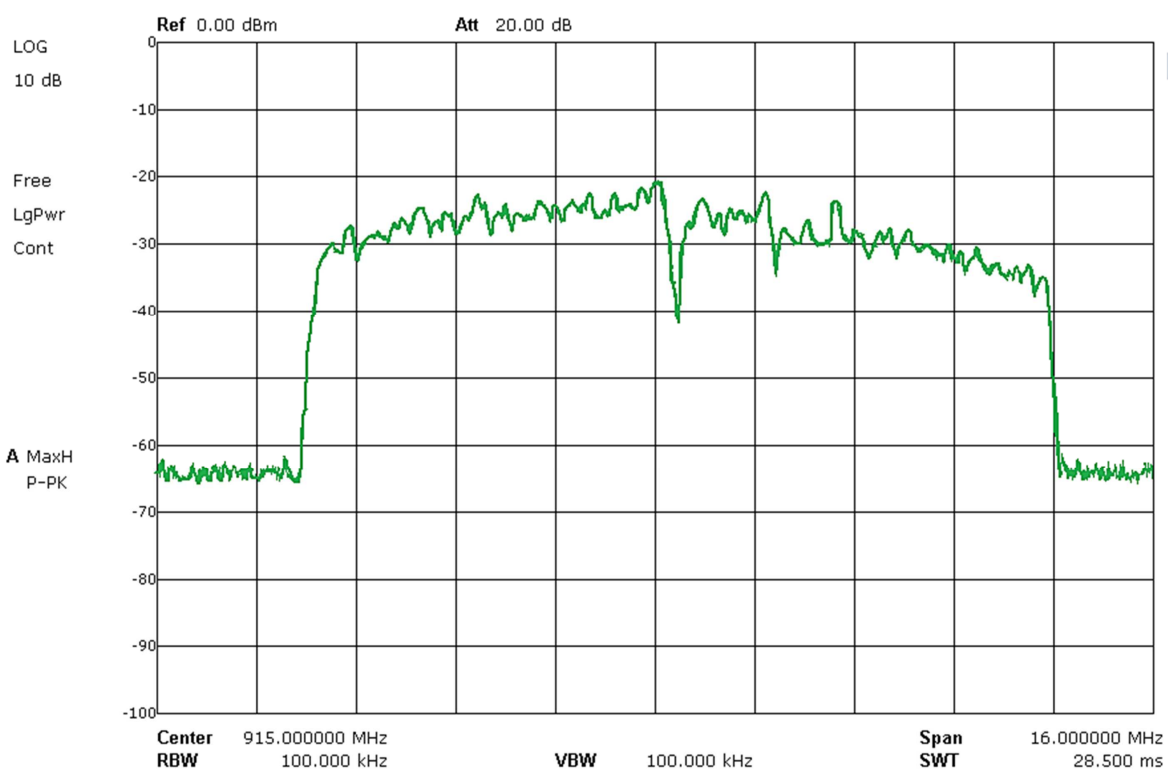

Fig. 16 The F-OFDM power spectrum with unequal sub-band sizes at $915 \mathrm{MHz}$

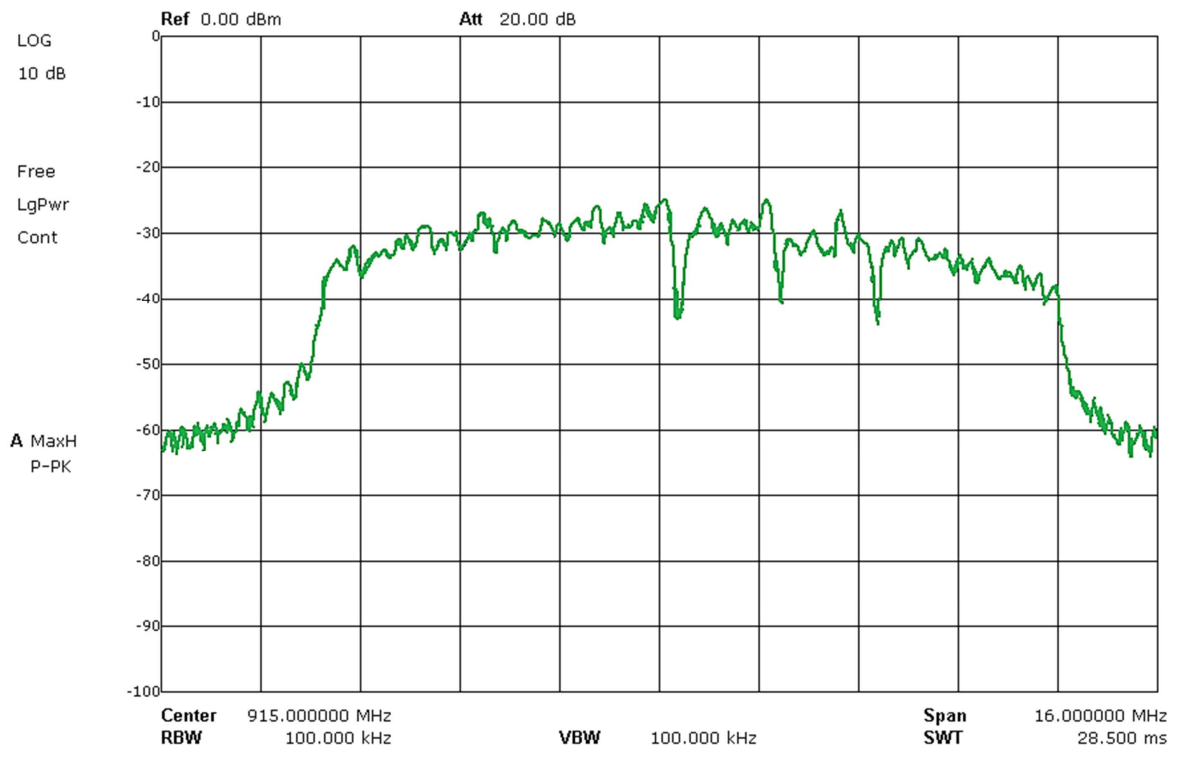

Fig. 17 The OFDM power spectrum with unequal sub-band sizes at $915 \mathrm{MHz}$

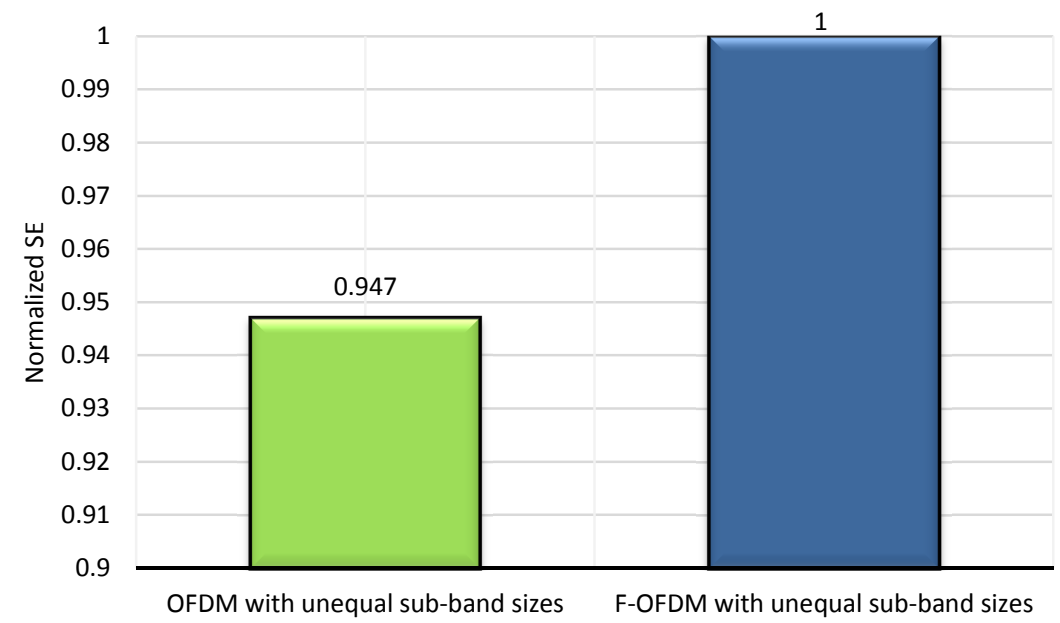

Fig. 18 Normalized SE for the F-OFDM with unequal sub-band sizes in practice 
Table 3 Comparison of the F-OFDM waveforms proposed in previous studies and this study

\begin{tabular}{|c|c|c|c|c|c|}
\hline Ref. & $\begin{array}{c}\text { Applied } \\
\text { filter }\end{array}$ & $\begin{array}{c}\text { Number of the } \\
\text { sub-band filtered }\end{array}$ & $\begin{array}{c}\text { BER } \\
\text { (compared to that of OFDM) }\end{array}$ & $\begin{array}{c}\text { SE enhancement } \\
\text { (compared to that of OFDM) }\end{array}$ & Used filter type \\
\hline$[4]$ & Per sub-band & 3 & Not specified & $7.47 \%$ higher & Window-sinc \\
\hline$[6]$ & Per user & 3 & Worse & Not specified & Soft truncation \\
\hline$[10]$ & Per sub-band & 3 & Same & Not specified & $\begin{array}{c}\text { Soft-truncated sinc } \\
\text { and equi-ripple }\end{array}$ \\
\hline$[13]$ & Per sub-band & 2 & Same & Same & Window-sinc \\
\hline$[14]$ & Per sub-band & 2 & Same & Higher & Whydyas \\
\hline$[15]$ & Per user & 2 & Better & Not specified & Window-sinc \\
\hline$[16]$ & Per sub-band & 2 & Better & $\begin{array}{c}6 \% \text { (in simulation) and } \\
5.8 \% \text { (in practice) higher }\end{array}$ & Window-sinc \\
\hline $\begin{array}{c}\text { This } \\
\text { work }\end{array}$ & Per sub-band & 4 & Approximately same & & \\
\hline
\end{tabular}

\section{Conclusions}

This study presents F-OFDM-based sub-band filtering as a waveform contender to meet the requirements of 5G applications in terms of SE by reducing the guard band and increasing the system capacity. F-OFDM divides the allocated spectrum into smaller sub-bands, and each sub-band performs a filter that has different filter specifications to maintain the orthogonality between the subcarriers in each sub-band. The proposed scenario splits the spectrum into unequal sub-band sizes. Compared to the SE using OFDM, the SE using F-OFDM is increased by $6 \%$ under the LabVIEW NXG simulation environment because of the reduction of the guard band between the sub-bands, and achieves 5.8\% under the real indoor environments based on the SDR technology. These percentages match the requirements of the $5 \mathrm{G}$ system for the allocated spectrum.

\section{Conflicts of Interest}

The authors declare no conflict of interest.

\section{References}

[1] S. Ahmadi, 5G NR: Architecture, Technology, Implementation, and Operation of 3GPP New Radio Standards, 1st ed., London: Academic Press, 2019.

[2] Huawei Technologies, "5G: New Air Interface and Radio Access Virtualization," https://www.huawei.com/minisite/has2015/img/5g_radio_whitepaper.pdf, April 2015.

[3] P. Banelli, S. Buzzi, G. Colavolpe, A. Modenini, F. Rusek, and A. Ugolini, "Modulation Formats and Waveforms for the Physical Layer of 5G Wireless Networks: Who Will Be the Heir of OFDM?” IEEE Signal Processing Magazine, vol. 31, no. 6, pp. 80-93, November 2014.

[4] J. Wang, A. Jin, D. Shi, L. Wang, H. Shen, D. Wu, et al., "Spectral Efficiency Improvement with 5G Technologies: Results from Field Tests," IEEE Journal on Selected Areas in Communications, vol. 35, no. 8, pp. 1867-1875, August 2017.

[5] S. K. Shaikhah and S. Mustafa, "A Robust Filter Bank Multicarrier System as a Candidate for 5G," Physical Communication, vol. 43, 101228, December 2020.

[6] J. Abdoli, M. Jia, and J. Ma, "Filtered OFDM: A New Waveform for Future Wireless Systems," IEEE 16th International Workshop on Signal Processing Advances in Wireless Communications, pp. 66-70, June 2015.

[7] M. Gupta, A. S. Kang, and V. Sharma, "Comparative Study on Implementation Performance Analysis of Simulink Models of Cognitive Radio Based GFDM and UFMC Techniques for 5G Wireless Communication,” Wireless Personal Communications, in press.

[8] A. Ijaz, L. Zhang, P. Xiao, and R. Tafazolli, “Analysis of Candidate Waveforms for 5G Cellular Systems," https://www.intechopen.com/chapters/52817, December 14, 2016.

[9] A. Yazar, M. Elkourdi, and H. Arslan, "Waveform Designs for Cognitive Radio and Dynamic Spectrum Access Applications,” https://link.springer.com/content/pdf/10.1007\%2F978-981-10-1389-8_3-1.pdf, 2019.

[10] X. Zhang, M. Jia, L. Chen, J. Ma, and J. Qiu, "Filtered-OFDM-Enabler for Flexible Waveform in the 5th Generation 
Cellular Networks,” IEEE Global Communications Conference, pp. 1-6, December 2015.

[11] “UHD," https:// kb.ettus.com/UHD, March 01, 2020.

[12] E. Grayver, Implementing Software Defined Radio, 1st ed., New York: Springer, 2012.

[13] L. Yang and Y. Xu, "Filtered-OFDM System Performance Research Based on Nuttall's Blackman-Harris Window," IEEE 17th International Conference on Communication Technology, pp. 687-691, October 2017.

[14] R. Gerzaguet, D. Demmer, J. B. Doré, and D. Ktiénas, "Block-Filtered OFDM: A New Promising Waveform for Multi-Service Scenarios,” IEEE International Conference on Communications, pp. 1-6, May 2017.

[15] M. Yang, Y. Chen, and L. Du, "Interference Analysis and Filter Parameters Optimization for Uplink Asynchronous F-OFDM Systems,” IEEE Access, vol. 7, pp. 48356-48370, April 2019.

[16] K. S. Chandran and C. K. Ali, "Filtered-OFDM with Index Modulation for Mixed Numerology Transmissions," 6th International Conference on Advanced Computing and Communication Systems, pp. 306-310, March 2020.

[17] “X300/X310,” https://kb.ettus.com/X300/X310\#X310_2, March 07, 2020.

[18] F. J. Harris, "On the Use of Windows for Harmonic Analysis with the Discrete Fourier Transform," Proc. of the IEEE, vol. 66, no. 1, pp. 51-83, January 1978.

Copyright $(\odot$ by the authors. Licensee TAETI, Taiwan. This article is an open access article distributed under the terms and conditions of the Creative Commons Attribution (CC BY-NC) license (https://creativecommons.org/licenses/by-nc/4.0/). 